\title{
A NEUROLOGICAL CATASTROPHE AFTER ANOTHER - CASE REPORT
}

\author{
Afonso Guilherme Schmidt ${ }^{1, \star}$, André Lucas Ribeiro ${ }^{1}$, Andrea Worm Furtado ${ }^{1}$, Augusto Emilio Hinterholz ${ }^{1}$, Larissa Martinelli \\ Dullius ${ }^{1}$, Larissa Vargas Cruz ${ }^{1}$, Charles Lubianca Kohem ${ }^{1}$, Vanessa Hax ${ }^{1}$, Natalia Sarzi Sartori ${ }^{1}$
}

1.Universidade Federal do Rio Grande do Sul, Porto Alegre (RS), Brazil.

*Corresponding author: afonsogschmidt@gmail.com

\section{BACKGROUND}

Hemorrhagic stroke (HS) in systemic lupus erythematosus (SLE) patients are two to three times more frequent than in healthy people, but are still uncommon events with high morbimortality. Various risk factors are implicated in the pathogenesis, such as the use of anticoagulants, vasculitis, hemorrhagic transformation of an ischemic stroke, and thrombocytopenia, the latter accounting for more than $50 \%$ of the cases. Similarly, transverse myelitis is a severe complication of the disease in about $2 \%$ of the patients and leads to permanent functional loss in approximately $50 \%$ of the cases. We report a case in which these two complications happened in the same patient, without major factors for HS identifiable.

\section{CASE REPORT}

A 25-year-old woman with a history of SLE since she was 11, with leukolymphopenia, skin and joint manifestations, without antiphospholipid antibody syndrome (APS), in remission with mycophenolate in slow dose reduction, developed longitudinal extensive transverse myelitis in most of cervical, thoracic and lumbar spinal cord seen in magnetic resonance imaging (MRI) (Fig. 1), with paraplegia and sensory level in T12. CSF with only increased protein, reduced serum complements. Intravenous methylprednisolone $1 \mathrm{~g}$ was instituted for 3 days, followed by $1.5 \mathrm{mg} / \mathrm{kg} / \mathrm{day}$. Seven days later, the patient suffered septic shock and loss of kidney function due to pneumonia; $72 \mathrm{~h}$ after that, she presents with an altered sensorium requiring intubation and intense blood pressure lability. On physical examination, she had fixed bilateral mydriasis, absent corneal reflex even after several hours without sedation, normal cardiopulmonary evaluation, light parameters of mechanical ventilation, normal coagulogram as well as hepatic function, lactate and electrolytes. Computerized tomography (CT) scan showed pontine hemorrhage (Fig. 2, arrival CT left, current CT right). Vascular malformations were ruled out, and there was no sign of central nervous system vasculitis in a cranial MRI. No new immunosuppressive measures were instituted in view of the active pulmonary infection. She evolved with progressive clinical improvement and was discharged from the hospital several weeks later, with paraplegy, right central facial palsy, and cardiac autonomic dysfunction, needing a pacemaker.
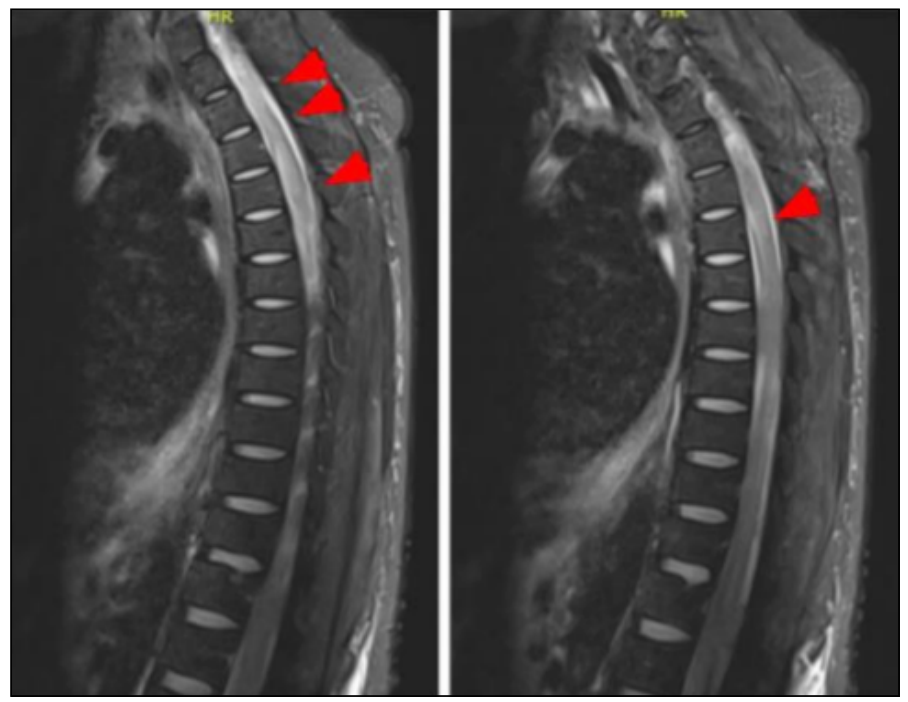

Figure 1. Magnetic resonance imaging showing longitudinal extensive transverse myelitis in most of cervical, thoracic and lumbar spinal cord.

Realização: 


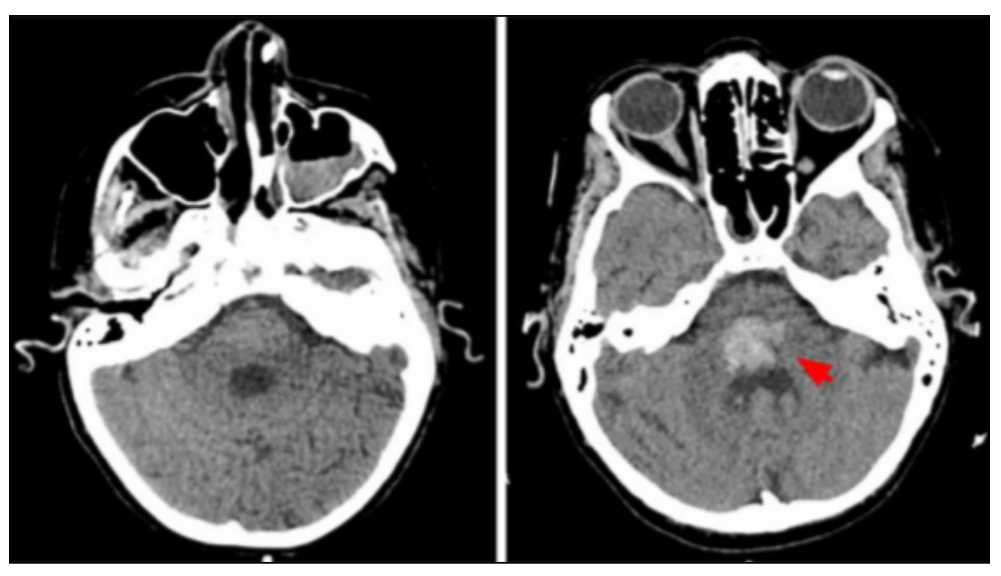

Figure 2. Computerized tomography scan showing pontine hemorrhage.

\section{CONCLUSION}

Several factors may be implicated in hemorrhagic stroke, one of which may be vessel wall weakness due to endothelial dysfunction leading to rupture. This seems to be the case in our patient, since the main alternative causes were ruled out. To the best of our knowledge, the association of these two serious but uncommon events in the same lupus patient has not been reported before.

\section{KEYWORDS}

Lupus, Stroke, Myelitis. 